\title{
Intra- and inter-observer reproducibility of multilayer cardiac magnetic resonance feature tracking derived longitudinal and circumferential strain
}

\author{
Saikrishna Ananthapadmanabhan ${ }^{1}$, Echo Deng ${ }^{1}$, Giuseppe Femia ${ }^{2}$, Simon Tang ${ }^{2}$, Eng-Siew Koh ${ }^{3}$, \\ Andreas Schuster ${ }^{4,5,6}$, Raj Puranik ${ }^{7}$, Pankaj Gupta ${ }^{7}$, Tuan Nguyen ${ }^{1,2}$, Hany Dimitri ${ }^{1,2}$, James Otton ${ }^{1,2}$ \\ ${ }^{1}$ Faculty of Medicine, University of New South Wales, Bossley Park, Sydney, NSW, Australia; ${ }^{2}$ Cardiology Department, ${ }^{3}$ Department of Radiation \\ Oncology, Liverpool Hospital, Liverpool, Sydney, Australia; ${ }^{4}$ Department of Cardiology, Royal North Shore Hospital, The Kolling Institute, \\ Northern Clinical School, University of Sydney, Sydney, Australia; ${ }^{5}$ University Medical Center Göttingen, Department of Cardiology and \\ Pneumology, Georg-August University, Göttingen, Germany; ${ }^{6}$ German Center for Cardiovascular Research (DZHK), Partner Site Göttingen, \\ Göttingen, Germany; ${ }^{7}$ Cardiology Department, Royal Prince Alfred Hospital, Newtown, Sydney, Australia \\ Contributions: (I) Conception and design: J Otton, H Dimitri, T Nguyen; (II) Administrative support: None; (III) Provision of study materials or \\ patients: J Otton, H Dimitri, T Nguyen, R Puranik, P Gupta, ES Koh, S Tang; (IV) Collection and assembly of data: S Ananthapadmanabhan, \\ G Femia, E Deng; (V) Data analysis and interpretation: S Ananthapadmanabhan; (VI) Manuscript writing: All authors; (VII) Final approval of \\ manuscript: All authors. \\ Correspondence to: Saikrishna Ananthapadmanabhan. Faculty of Medicine, University of New South Wales, Sydney, NSW 2052, Australia. Email: \\ saikrishnaanantha96@gmail.com.
}

Background: Multilayer strain measurement with cardiac magnetic resonance feature tracking (CMRFT) allows independent assessment of endocardial and epicardial strain. This novel method of layer-specific quantification of myocardial deformation parameters provides greater insight into contractility compared to whole-layer strain analysis. The clinical utility of this technique is promising. The aim of this study is to investigate the intra- and inter- observer reproducibility of CMR-FT derived multilayer global longitudinal strain (GLS) and global circumferential strain (GCS) parameters in the setting of normal cardiac function, cardiac pathology, and differing MRI field strengths.

Methods: We studied 4 groups of 20 subjects, comprising of patients with dilated cardiomyopathy, ischemic heart disease, and patients without cardiac pathology at both 1.5 and 3 T. Quantitative measures of wholelayer and multi-layer longitudinal and circumferential strain were calculated using CMR-FT software.

Results: Intraclass correlation coefficients (ICC) for intraobserver reproducibility of endocardial, epicardial, and whole-layer measurements of GLS were 0.979, 0.980, and 0.978 respectively, and those for GCS were $0.986,0.977$, and 0.985 . ICCs for inter-observer reproducibility of endocardial, epicardial, and whole-layer measurements of GLS were 0.976, 0.970, and 0.976, and those for GCS were 0.982, 0.969, and 0.981. Bland Altman analysis showed minimal bias and acceptable limits of agreement (LOA) within each patient subgroup and the overall cohort. Circumferential and longitudinal strain parameters were equally reproducible in the overall cohort.

Conclusions: CMR-FT derived multilayer measurements of longitudinal and circumferential strain demonstrate high intra- and inter- observer reproducibility, with suitability for use in clinical practice.

Keywords: Cardiac magnetic resonance (CMR); feature tracking (FT); multilayer; reproducibility

Submitted Nov 22, 2019. Accepted for publication Dec 24, 2019.

doi: $10.21037 /$ cdt.2020.01.10

View this article at: http://dx.doi.org/10.21037/cdt.2020.01.10 


\section{Introduction}

Cardiovascular magnetic resonance (CMR) is increasingly used for the evaluation and routine management of patients with coronary artery disease and cardiomyopathies. Strain imaging permits analysis of myocardial deformation, described with respect to the directional component of motion-radial, circumferential, or longitudinaland is an important parameter for investigating cardiac function (1). The assessment of deformation parameters has emerged as an objective and accurate clinical tool in analysing contractility associated with various cardiac pathologies, with echocardiographic studies demonstrating greater sensitivity to functional impairment and providing additional prognostic value compared to conventional parameters, namely left ventricular ejection fraction (LVEF) (2-4).

CMR feature tracking (CMR-FT) has been introduced as a recent advancement of tissue tracking technology and is a fast, robust technique for the quantification of myocardial strain by offline analysis of standard CMR cine steady-state free precession images $(1,5,6)$. It is a desirable alternative to CMR myocardial tagging, whose clinical feasibility is undermined by a somewhat elaborate acquisition procedure, the requirement for additional CMR scans, and intensive post-processing. CMR-FT is similar to speckle tracking echocardiography (STE) and incorporates border tracking of the endocardium and epicardium and pattern tracking of anatomical features and intra-myocardial tissue voxel motion (5). CMR-FT derived single-layer measurements for global longitudinal strain (GLS) (7) and global circumferential strain (GCS) $(7,8)$ have been validated against CMR myocardial tagging, the gold standard of quantitative strain analysis.

Novel advancements in CMR-FT software have facilitated multilayer or layer-specific strain analysis, allowing independent assessment of global myocardial mechanics at the endocardial and epicardial levels (9). Many cardiac pathologies differ in their effects on myocardial tissue at different levels (10-12). For example, non-ST elevation myocardial infarction predominantly affects subendocardial tissue (13). Myocarditis may affect epicardial layers, particularly in the inferolateral wall (14). Analysis of strain specific to myocardial layer has strong potential for utility in a variety of clinical conditions where localisation of pathology may help establish the extent and nature of the underlying pathology.
While, CMR-FT is now widely utilised, multilayer strain has only recently been developed and requires detailed evaluation before it can be applied to research and clinical applications. This study aims to investigate the intraobserver and inter-observer reproducibility of multilayer CMRFT in both healthy volunteers and in patients with cardiac pathology.

\section{Methods}

\section{Subject recruitment}

Ethics approval for this study was obtained from the Concord Human Research Ethics Committee.

Cardiac MRI scans from four cohorts each consisting of 20 subjects were analysed. The first cohort consisted of patients diagnosed with a dilated cardiomyopathy (DCM), where all patients had a global reduction in ejection fraction with ischemic injury excluded on coronary angiography and late gadolinium enhancement imaging. A second patient cohort consisted of patients scanned within 7 days of ST elevation myocardial infarction. These patients were recruited using standard electrocardiography (ECG) criteria acute ST elevated myocardial infarction (presence of ST elevation in 2 contiguous leads $\geq 2 \mathrm{~mm}$ for leads $\mathrm{V} 1-\mathrm{V} 3$, or $\geq 1 \mathrm{~mm}$ for other leads or for posterior MI $\geq 1 \mathrm{~mm}$ ST depression for leads V2-V3) treated by primary percutaneous coronary intervention (PCI), thrombolysis or rescue PCI. All patients had a verified total occlusion or severe stenosis after reperfusion of a major epicardial vessel on coronary angiography. The third and fourth groups consisted of normal controls, with an absence of a history or clinical evidence of cardiovascular disease. One control group was scanned at $1.5 \mathrm{~T}$ while the other was scanned at $3 \mathrm{~T}$ to enable assessment of reproducibility at differing field strengths.

Scans demonstrating cardiac pathology were collated from consecutive cases of clinically referred subjects undergoing a cardiac MRI. Control scans were obtained from normal volunteers or from patients with early breast cancer in whom previous cardiac pathology was excluded, and in whom scanning was performed prior to any adjuvant treatment. Standard clinical exclusion criteria included patients with end stage renal failure, allergy to Gadolinium contrast, prior valvular cardiothoracic surgery or congenital heart disease, and other MRI exclusions, and age $<18$ or $>$ 85 years. 


\section{CMR acquisition}

All CMR measurements were performed in a standard supine position using commercially available machines. DCM and ischemic heart disease cohorts were scanned at 1.5 T (Siemens Symphony). Control groups were scanned at both $1.5 \mathrm{~T}$ (Philips Achieva) or $3 \mathrm{~T}$ (Siemens Skyra). Images were acquired at $8-\mathrm{mm}$ slice thickness with a typical in-plane resolution of $1.5625 \mathrm{~mm} \times 1.5625 \mathrm{~mm}$ and 25 phases per cardiac cycle. A multi-technique imaging protocol was implemented with an ECG-gated steady state free-precession cine sequence taken during periods of breath holding taken in the following planes: left ventricular (LV) 2-chamber, 3-chamber, and 4-chamber in the long axis plane and equidistant short-axis planes completely covering both ventricles and including basal, mid-ventricular, and apical segments. Each subject had a complete data set with short-axis stack and 3 long-axis views. An example of representative CMR feature tracking is shown in Figure 1.

\section{CMR-FT analysis}

Quantitative measurements of GLS and GCS in whole, endocardial, and epicardial layers were performed by offline analysis of cine CMR images using commercially available, dedicated feature tracking software (cvi42, Circle, Calgary, Canada, version 5.5). The LV endocardial and epicardial borders were manually delineated in the enddiastolic phase in all short- and long- axes slices where the $L V$ myocardium is visible and intact. The upper septal insertion point of the right ventricle (RV) was defined in the shot-axis series as an anatomic landmark to allow accurate segmentation of the $\mathrm{LV}$ according to a recognised standard model. The extent of the LV myocardium was defined in the long-axis series to define the analysis range. All assessors analysed the same slices for each subject in the short-axis stack. Manual contours were adjusted if there was evidence of poor tracking, whereby the contours deviated from the endocardial and epicardial borders based on visual judgement.

GLS was derived from 3 long axis views (2-chamber, 3-chamber, and 4-chamber). GCS was measured from 3 parasternal short axis views (basal, mid-ventricular, and apical).

Endocardial and epicardial GLS were calculated by averaging the peak endocardial and epicardial longitudinal strain values, respectively, from the 2-chamber, 3-chamber, and 4-chamber slices of the long-axis cine images. Endocardial and epicardial GCS were calculated using an identical approach in the short-axis series.

For the evaluation of intraobserver reproducibility, the strain analysis was repeated by the main assessor (Assessor 1) for all scans $(\mathrm{n}=80)$ using the same stored acquisition data, with a time interval of 2 weeks between both measurements. For the evaluation of inter-observer reproducibility, two other assessors (Assessor 2 and Assessor 3) were involved, with Assessor 2 analysing the ST-elevation myocardial infarction (STEMI), Controls 3-T, and DCM cohorts $(\mathrm{n}=60)$ and Assessor 3 analysing the Controls 1.5-T cohort $(n=20)$. Assessor 1 and 2 are medical students from the University of New South Wales who received training on using the feature tracking software. This included reading the vendor handbook, watching tutorial videos, and training with, receiving instruction from, and demonstrating proficiency in strain analysis to a cardiac imaging specialist. Assessor 3 is a cardiology registrar working at Liverpool Hospital. All three assessors used the same technique of manually delineating endocardial and epicardial contours for strain analysis. The calculations of endocardial and epicardial strain was only performed after each scan had been analysed thrice; twice by Assessor 1 and once by Assessors 2 or 3 . This ensured that Assessor 1 was blinded to their previous measurements, and that Assessors 2 and 3 were blinded to Assessor 1's results at the time of analysis.

\section{Statistical analysis}

All statistical analysis was performed using commercially available software, IBM SPSS Statistics 25 (SPSS Inc., Chicago, Illinois, USA). A $\mathrm{P}<0.05$ was considered statistically significant. Continuous data is expressed as mean \pm standard deviation. Evaluation of intra and interobserver reproducibility was done using two-way mixedeffect intraclass correlation coefficients for absolute agreement. Agreement was measured using Bland Altman plots. Comparison of agreement was analysed using Pearson's correlation coefficient and Fisher r-to-z transformation, with an absolute $\mathrm{z}$-observed value $>1.96$ and $\mathrm{P}<0.05$ considered statistically significant.

\section{Results}

\section{Intra- and inter-observer reproducibility of CMR-FT derived multilayer GLS and GCS}

Overall there was excellent intra- and inter-observer reproducibility in all multilayer parameters with results 
demonstrating similar consistency between longitudinal and circumferential strain parameters. The ICC values for intraobserver reproducibility for endocardial, epicardial, and whole-layer GLS were $0.979,0.980$, and 0.978 respectively, and for GCS were $0.986,0.977$, and 0.985 . The ICC values for inter-observer reproducibility for endocardial, epicardial, and whole-layer GLS were 0.976, 0.970, and 0.976, respectively, and for GCS were 0.982, 0.969, and 0.981. Bland Altman analysis revealed minimal bias between the two measurements for both intraobserver and interobserver reproducibility and showed good to excellent agreement within narrow limits of agreement, as shown in Tables 1,2. Bland Altman plots for intra- and inter-observer agreement are shown in Figures 2 and 3 respectively

A data supplement (available online: http://cdn.amegroups. $\mathrm{cn} /$ static/application/802a898c32fc1c0a8623752a6b923c66/ cdt.2020.01.10-1.pdf) provides Bland Altman plots of intraand inter-observer reproducibility for each individual cohort.

Demographic data of individual and total cohorts is shown in Table 3.

\section{Discussion}

The main finding of the current study is that CMR-FT derived measurements of multilayer strain have excellent intraobserver and inter-observer reproducibility in a mixed cohort of healthy individuals and subjects with cardiac disease, both for longitudinal and circumferential deformation parameters.

Previous studies investigating whole-layer global strain values have shown that the reproducibility of CMR-FT is similar to that of CMR myocardial tagging, the reference standard for strain assessment, for GLS (15) and GCS $(7,15,16)$. CMR-FT derived strain values demonstrate less variability when compared to STE, due to the former's superior image quality and latter's issues with ultrasound dropout, reverberations, and increased field noise (5). The tomographic nature of CMR is beneficial for reproducibility, allowing strain assessment to be performed using identical myocardial slices. Earlier CMR-FT studies reported higher variability for GLS compared to GCS (16), with coefficient of variation of $12.3 \mathrm{vs} .2 .8$ for intraobserver agreement and 10.9 vs. 4.9 for inter-observer agreement. This problem may likely be attributable to deterioration of reproducibility at the apex, possible difficulty in manual contouring at the blood-tissue interface, and a suspected tendency to track the non-contractile mitral valve apparatus (5). However, our results show near identical reproducibility for multilayer circumferential and longitudinal strain.

Our results showed that endocardial, epicardial, and whole-layer GLS were more reproducible in the subjects with cardiac pathology compared to the healthy controls for both intraobserver and inter-observer measurements. This finding is partially attributable to encountered difficulties in manual contouring in the long-axis of the healthy control cohort, leading to smaller correlation coefficients when compared to the cardiac disease cohorts. It should also be noted that the intra-class correlation reproducibility metric is inflated by matched extreme values, which are more commonly found in the pathological cohort. Normal volunteers had increased annular motion and more through plane motion of the papillary muscles. Quantification may also have been affected by image quality. We can speculate but we are unable to definitively state the exact cause. We therefore present the data as collected.

Heinke et al. (17) described a post-processing standardisation of deriving CMR-FT GLS in which assessors evaluated the quality of contour placement following manual delineation and performed adjustments as necessary based on evidence of poor tracking. This approach was shown to improve intra- and inter-observer agreement when comparing pre- and post-standardisation data. In our study, we used a similar, yet more informal, approach to manual contouring. Furthermore, when considering the entire cohort $(n=80)$, our results show excellent intra and inter-observer agreement despite the difference in experience between the three assessors.

\section{Clinical implications}

Our results demonstrate that inter-observer reproducibility is similar to intraobserver reproducibility for all parameters of multilayer GLS and GCS. This, in conjunction with the high ICC values, indicates that subtle changes in endocardial and epicardial strain can be determined using serial examinations with high accuracy and reliability, especially in clinical settings where scans may be analysed by multiple clinicians of varying skill levels and experience.

A layer-specific approach to strain imaging may establish new diagnostic methods for evaluating cardiac function that are unavailable with whole-layer strain analysis or conventional echocardiographic parameters such as LVEF. The inner subendocardial fibres are affected earlier, and to a greater extent, in the course of disease, and have superior diagnostic utility in identifying coronary artery disease compared to LVEF (13). Multilayer circumferential strain 


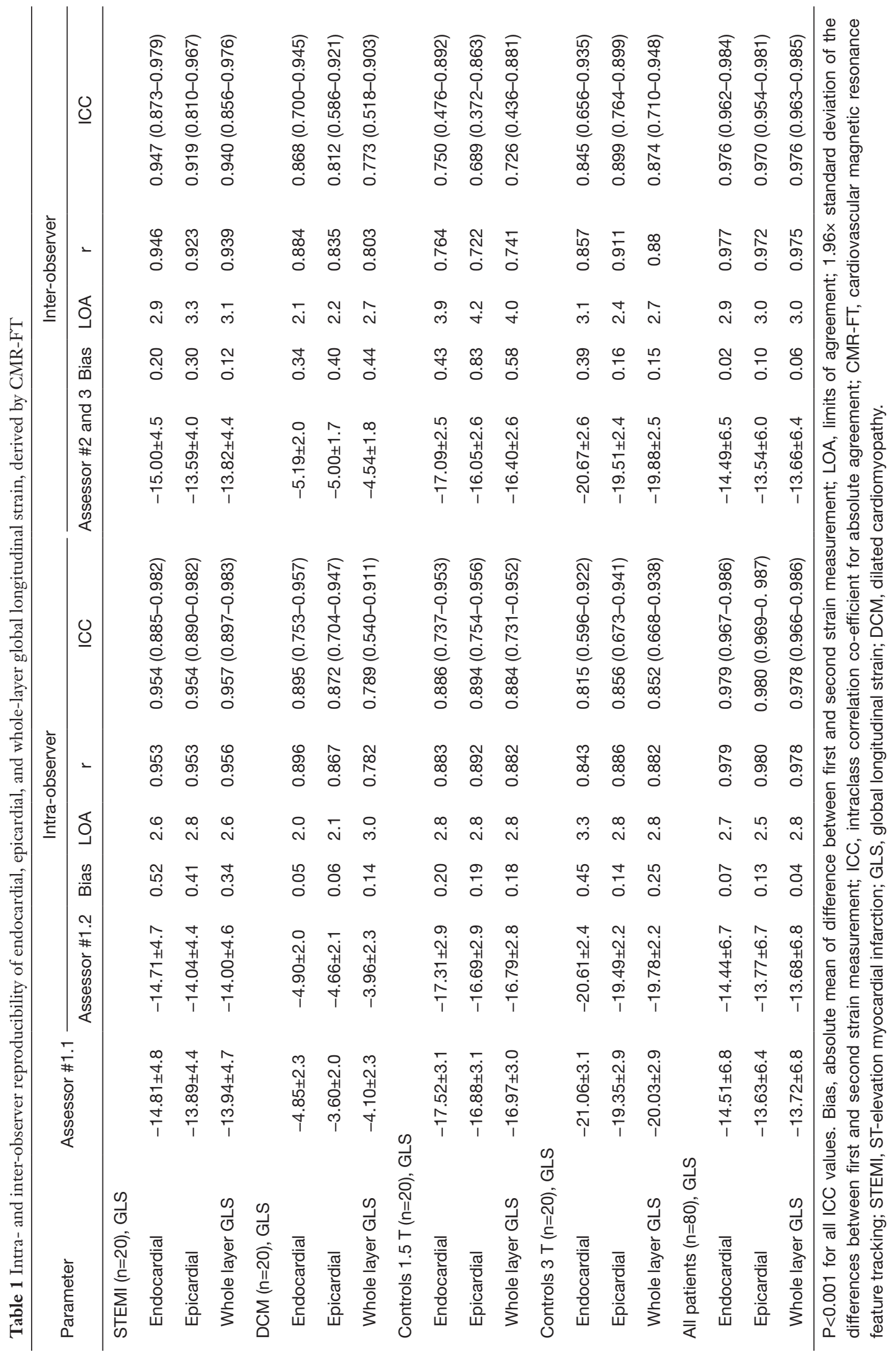




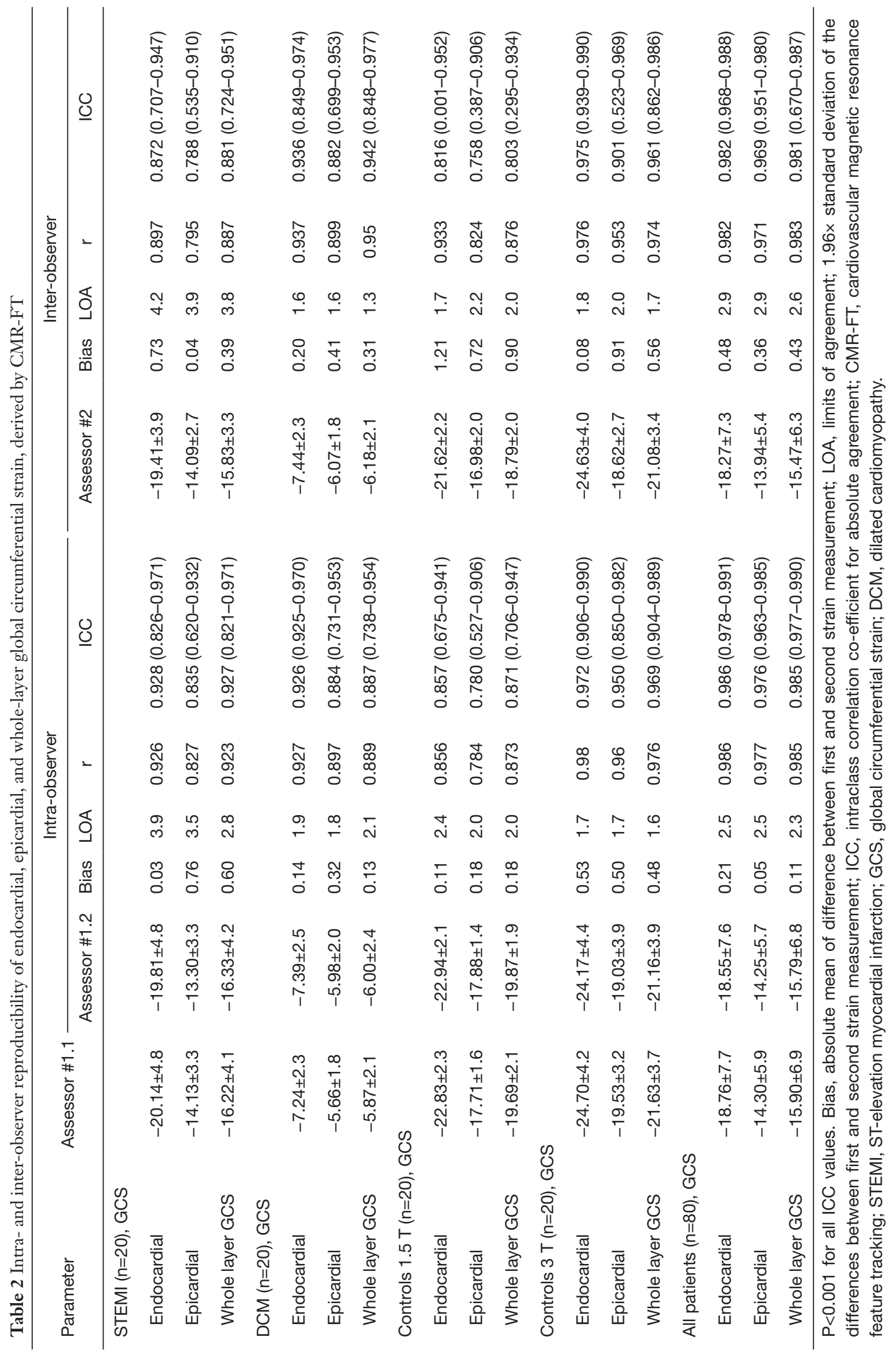


Table 3 Baseline demographics of individual and total cohorts

\begin{tabular}{lcccc}
\hline Variable & STEMI $(\mathrm{n}=20)$ & DCM $(\mathrm{n}=20)$ & Controls $(\mathrm{n}=40)$ & Total cohort $(\mathrm{n}=80)$ \\
\hline Male gender $(\%)$ & 70.0 & 85.0 & 35.0 & 56.3 \\
Age, years & $56.7 \pm 8.3$ & $53.9 \pm 13.5$ & $54.2 \pm 13.7$ & $54.7 \pm 12.4$ \\
LVEF (\%) & $49.2 \pm 9.2$ & $19.2 \pm 6.4$ & $61.6 \pm 8.7$ & $43.4 \pm 19.7$ \\
\hline
\end{tabular}

STEMI, ST-elevation myocardial infarction; DCM, dilated cardiomyopathy; LVEF, left ventricular ejection fraction.
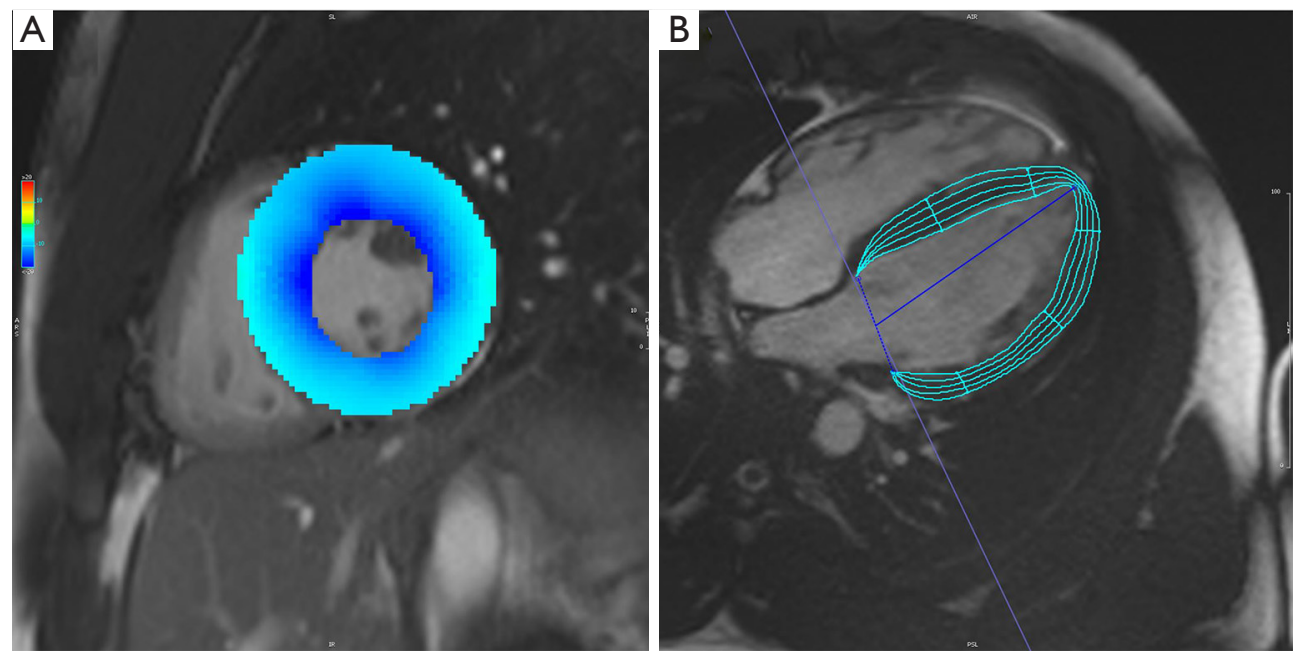

Figure 1 Representative CMR feature tracking, showing circumferential colour-coded strain in short-axis view (A) and long-axis 4 chamber strain with layer segmentation (B). CMR, cardiac magnetic resonance.

analysis by STE has demonstrated additional value in assessing infarct transmurality $(12,16)$, myocardial viability $(11,16)$, and estimating functional recovery in ischaemic heart disease (18-20). The value of layer-specific segmental strain measurement is unknown, with Ünlü reporting that endocardial and midwall segmental longitudinal strain measured by CMR does not improve detection of regional functional abnormalities (21). Our paper focusses only on the reproducibility of layer-specific global strain values.

CMR is being utilised to a greater extent in clinical practice in cardiology and strain analysis using CMRFT may play an important role in the diagnosis and management of cardiac disease. The high reproducibility reported in this study suggests that CMR-FT derived multilayer strain values are reliable for tracking changes in contractile function. The current literature on multilayer strain imaging is limited as it is a new technique, with studies focusing predominantly on STE. CMR-FT and STE have shown good inter-modality agreement for wholelayer strain imaging (22-25), yet a comparison on multilayer strain is needed. Future studies also need to investigate whether multilayer CMR-FT has similar diagnostic and prognostic implications to multilayer STE.

\section{Limitations}

This study has some limitations. Firstly, we performed strain analysis using only a single software version. Hence, we could not compare data with other commercially available CMR-FT software. Previous studies have investigated the inter-vendor reproducibility of single-layer strain and efforts are needed to harmonise analysis protocols to reduce the variability introduced by different software (26,27). Furthermore, Ünlü et al. reported significant intervendor variability in layer-specific segmental measurements of endocardial and midwall longitudinal strain. Second, currently available software is limited to two myocardial layers. Third, we believe that future studies should look at the intraobserver and inter-observer reproducibility of measuring the change in strain parameters between serial examinations, 


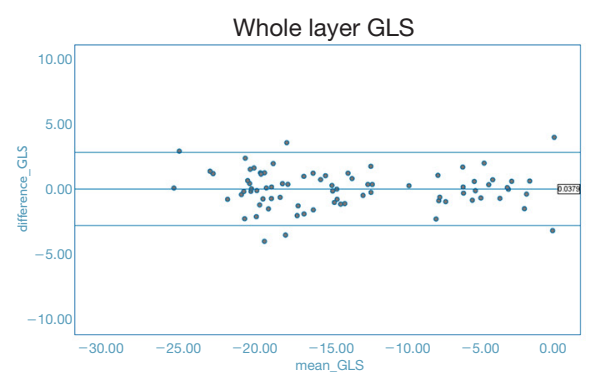

Bias $=0.04$, LOA $=2.8(2.8 ;-2.8)$

X-axis: mean GLS; Y-axis: difference GLS

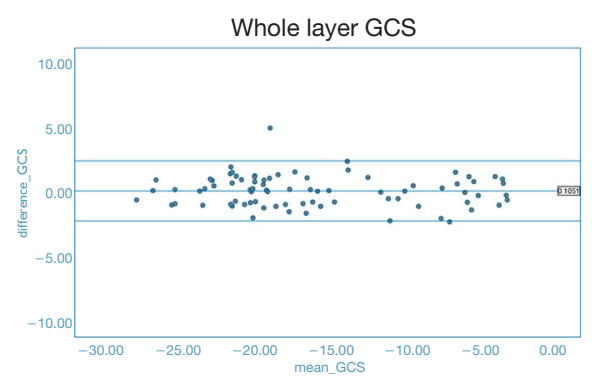

Bias $=0.11$, LOA $=2.3(2.4 ;-2.2)$

X-axis: mean GCS; Y-axis: difference GCS

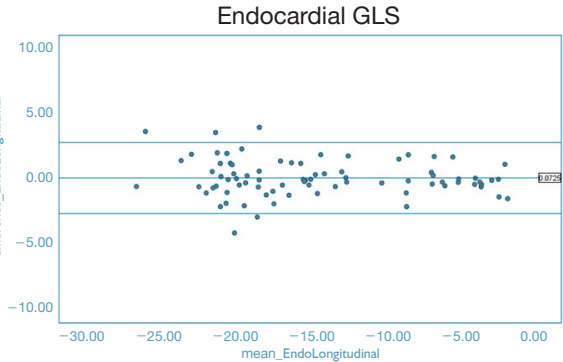

Bias $=0.07$, LOA $=2.7(2.8 ;-2.7)$

$X$-axis: mean endolongitudinal;

Y-axis: difference endolongitudinal

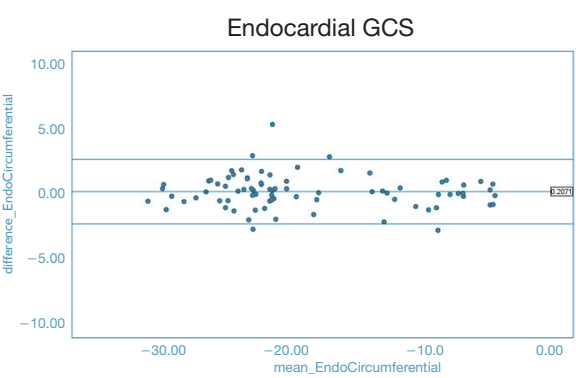

Bias $=0.21$, LOA $=2.5(2.7 ;-2.3)$

$\mathrm{X}$-axis: mean endocircumferential;

$Y$-axis: difference endocircumferential
Epicardial GLS

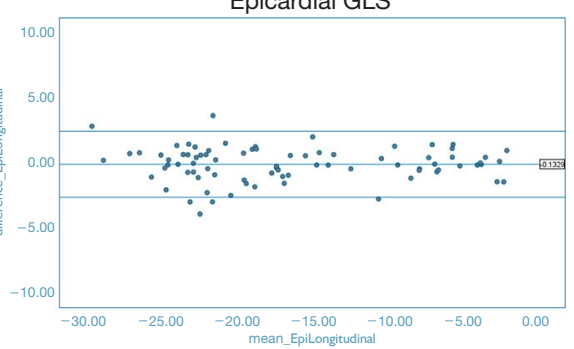

Bias $=0.13$, LOA $=2.5(2.4 ;-2.7)$

$X$-axis: mean epilongitudinal;

Y-axis: difference epilongitudinal

Epicardial GCS

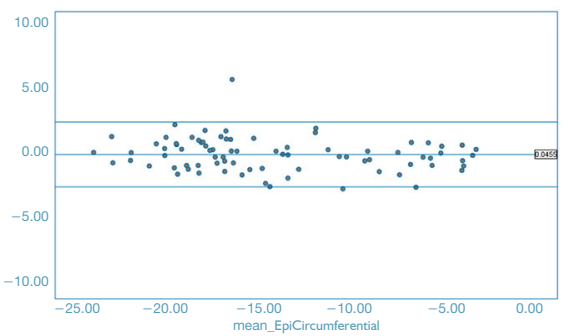

Bias $=0.05$, LOA $=2.5(2.6 ;-2.5)$

$X$-axis: mean epicircumferential;

Y-axis: difference epicircumferential

Figure 2 Bland Altman plots showing intra-observer agreement for CMR-FT derived multilayer GLS and GCS, in all subjects (n=80). GLS, global longitudinal strain; LOA, limits of agreement; GCS, global circumferential strain; CMR-FT, cardiovascular magnetic resonance feature tracking.

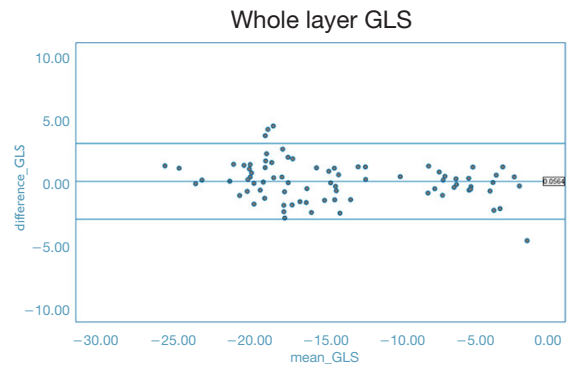

Bias $=0.06$, LOA $=3.0(3.1 ;-3.0)$

$X$-axis: mean GLS; Y-axis: difference GLS

Whole layer GCS

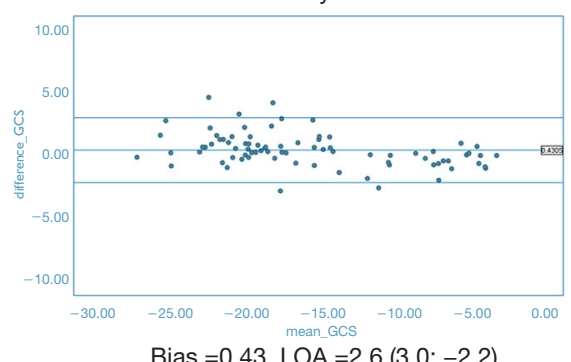

X-axis: mean GCS; Y-axis: difference GCS

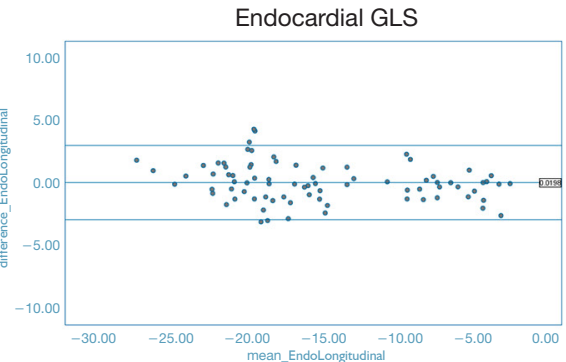

Bias $=0.02$, LOA $=2.9(2.9 ;-2.9)$

$X$-axis: mean endolongitudinal;

Y-axis: difference endolongitudinal

Endocardial GCS

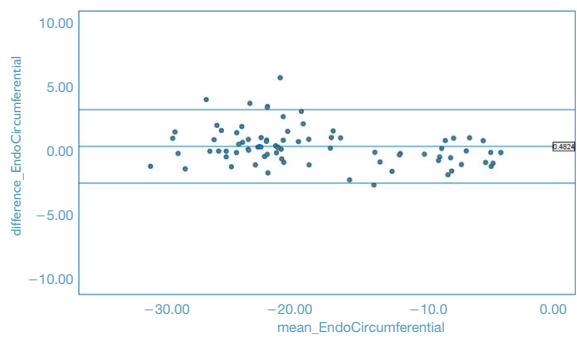

Bias $=0.48$, LOA $=2.9(3.4 ;-2.4)$

$X$-axis: mean endocircumferential;

Y-axis: difference endocircumferential

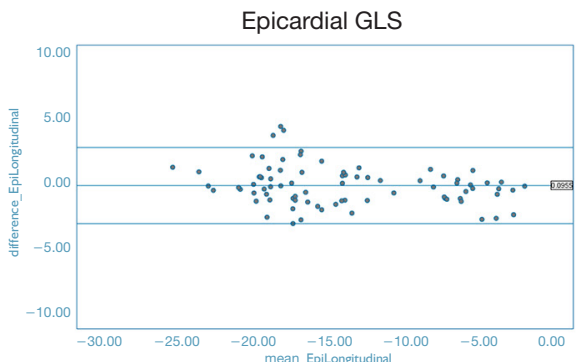

Bias $=0.10$, LOA $=3.0(3.1 ;-2.9)$

$\mathrm{X}$-axis: mean epilongitudinal;

Y-axis: difference epilongitudinal

Epicardial GCS

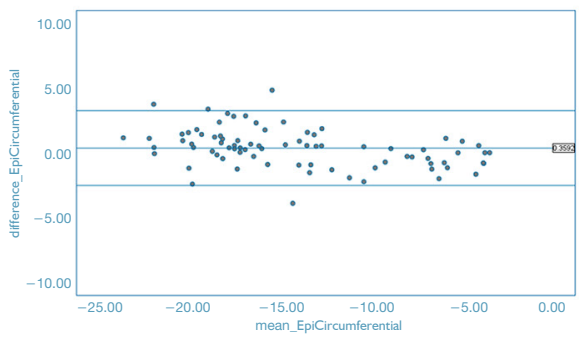

Bias $=0.36$, LOA $=2.9(3.3 ;-2.6)$

$\mathrm{X}$-axis: mean epicircumferential;

Y-axis: difference epicircumferential

Figure 3 Bland Altman plots showing inter-observer agreement for CMR-FT derived multilayer GLS and GCS, in all subjects (n=80). GLS, global longitudinal strain; LOA, limits of agreement; GCS, global circumferential strain. 
to assess the clinical utility of CMR-FT in monitoring cardiac function in patients with heart disease. Finally, further studies are necessary to investigate whether CMR-FT derived strain values are comparable despite differences in image acquisition procedures that commonly occur in clinical practice, such as sequence parameters and scanner hardware.

\section{Conclusions}

CMR-FT demonstrates excellent reproducibility for endocardial, epicardial, and whole-layer measurements of longitudinal and circumferential strain.

\section{Acknowledgments}

Funding: None.

\section{Footnote}

Conflicts of Interest: All authors have completed the ICMJE uniform disclosure form (available at http://dx.doi. org/10.21037/cdt.2020.01.10). The authors have no conflicts of interest to declare.

Ethical Statement: The authors are accountable for all aspects of the work in ensuring that questions related to the accuracy or integrity of any part of the work are appropriately investigated and resolved. This study was performed retrospectively and received ethics approval from the Concord Human Research Ethics Committee (HREC/11/CRGH/224). Written consent was obtained from subjects in the STEMI and DCM cohort. Consent was not required for subjects in the Controls 1.5- and 3-T cohort, as approved by the IRB.

Open Access Statement: This is an Open Access article distributed in accordance with the Creative Commons Attribution-NonCommercial-NoDerivs 4.0 International License (CC BY-NC-ND 4.0), which permits the noncommercial replication and distribution of the article with the strict proviso that no changes or edits are made and the original work is properly cited (including links to both the formal publication through the relevant DOI and the license). See: https://creativecommons.org/licenses/by-nc-nd/4.0/.

\section{References}

1. Claus P, Omar AMS, Pedrizzetti G, et al. Tissue Tracking
Technology for Assessing Cardiac Mechanics: Principles, Normal Values, and Clinical Applications. JACC Cardiovasc Imaging 2015;8:1444-60.

2. Kalam K, Otahal P, Marwick T. Prognostic implications of global LV dysfunction: a systematic review and metaanalysis of global longitudinal strain and ejection fraction. Heart 2014;100:1673-80.

3. Eitel I, Stiermaier T, Lange T, et al. Cardiac Magnetic Resonance Myocardial Feature Tracking for Optimized Prediction of Cardiovascular Events Following Myocardial Infarction. JACC Cardiovasc Imaging 2018;11:1433-44.

4. Romano S, Judd RM, Kim RJ, et al. Feature-Tracking Global Longitudinal Strain Predicts Death in a Multicenter Population of Patients With Ischemic and Nonischemic Dilated Cardiomyopathy Incremental to Ejection Fraction and Late Gadolinium Enhancement. JACC Cardiovasc Imaging 2018;11:1419-29.

5. Pedrizzetti G, Claus P, Kilner P, et al. Principles of cardiovascular magnetic resonance feature tracking and echocardiographic speckle tracking for informed clinical use. J Cardiovasc Magn Reson 2016;18:51.

6. Schuster A, Hor KN, Kowallick JT, et al. Cardiovascular Magnetic Resonance Myocardial Feature Tracking: Concepts and Clinical Applications. Circ Cardiovasc Imaging 2016;9:e004077.

7. Hor KN, Gottliebson WM, Carson C, et al. Comparison of magnetic resonance feature tracking for strain calculation with harmonic phase imaging analysis. JACC Cardiovasc Imaging 2010;3:144-51.

8. Moody WE, Taylor RJ, Edwards NC, et al. Comparison of magnetic resonance feature tracking for systolic and diastolic strain and strain rate calculation with spatial modulation of magnetization imaging analysis. J Magn Reson Imaging 2015;41:1000-12.

9. Adamu U, Schmitz F, Becker M, et al. Advanced speckle tracking echocardiography allowing a three-myocardial layer-specific analysis of deformation parameters. Eur J Echocardiogr 2009; 10:303-8.

10. Becker M, Altiok E, Lente C, et al. Layer-specific analysis of myocardial function for accurate prediction of reversible ischaemic dysfunction in intermediate viability defined by contrast-enhanced MRI. Heart 2011;97:748-56.

11. Becker M, Ocklenburg C, Altiok E, et al. Impact of infarct transmurality on layer-specific impairment of myocardial function: a myocardial deformation imaging study. Eur Heart J 2009;30:1467-76.

12. Altiok E, Neizel M, Tiemann S, et al. Layer-specific analysis of myocardial deformation for assessment of 
infarct transmurality: comparison of strain-encoded cardiovascular magnetic resonance with 2D speckle tracking echocardiography. Eur Heart J Cardiovasc Imaging 2013;14:570-8.

13. Sarvari SI, Haugaa KH, Zahid W, et al. Layer-specific quantification of myocardial deformation by strain echocardiography may reveal significant CAD in patients with non-ST-segment elevation acute coronary syndrome. JACC Cardiovasc Imaging 2013;6:535-44.

14. Friedrich MG, Sechtem U, Schulz-Menger J, et al. Cardiovascular magnetic resonance in myocarditis: A JACC White Paper. J Am Coll Cardiol 2009;53:1475-87.

15. Khan JN, Singh A, Nazir SA, et al. Comparison of cardiovascular magnetic resonance feature tracking and tagging for the assessment of left ventricular systolic strain in acute myocardial infarction. Eur J Radiol 2015;84:840-8.

16. Augustine D, Lewandowski AJ, Lazdam M, et al. Global and regional left ventricular myocardial deformation measures by magnetic resonance feature tracking in healthy volunteers: comparison with tagging and relevance of gender. J Cardiovasc Magn Reson 2013;15:8.

17. Heinke R, Pathan F, Le M, et al. Towards standardized postprocessing of global longitudinal strain by feature tracking - OptiStrain CMR-FT study. BMC Cardiovasc Disord 2019;19:267.

18. Woo JS, Kim WS, Yu TK, et al. Prognostic value of serial global longitudinal strain measured by two-dimensional speckle tracking echocardiography in patients with STsegment elevation myocardial infarction. Am J Cardiol 2011;108:340-7.

19. Huttin O, Coiro S, Selton-Suty C, et al. Prediction of Left Ventricular Remodeling after a Myocardial Infarction: Role of Myocardial Deformation: A Systematic Review and Meta-Analysis. PLoS One 2016;11:e0168349.

20. Shetye A, Nazir S, Squire I, et al. Global myocardial strain assessment by different imaging modalities to predict outcomes after ST-elevation myocardial infarction: A

Cite this article as: Ananthapadmanabhan S, Deng E, Femia G, Tang S, Koh ES, Schuster A, Puranik R, Gupta P, Nguyen T, Dimitri H, Otton J. Intra- and inter-observer reproducibility of multilayer cardiac magnetic resonance feature tracking derived longitudinal and circumferential strain. Cardiovasc Diagn Ther 2020;10(2):173-182. doi: 10.21037/cdt.2020.01.10 systematic review. World J Cardiol 2015;7:948-60.

21. Ünlü S, Mirea O, Pagourelias ED, et al. Layer-Specific Segmental Longitudinal Strain Measurements: Capability of Detecting Myocardial Scar and Differences in Feasibility, Accuracy, and Reproducibility, Among Four Vendors A Report From the EACVI-ASE Strain Standardization Task Force. J Am Soc Echocardiogr 2019;32:624-632.e11.

22. Obokata $M$, Nagata $Y, W u$ VC, et al. Direct comparison of cardiac magnetic resonance feature tracking and 2D/3D echocardiography speckle tracking for evaluation of global left ventricular strain. Eur Heart J Cardiovasc Imaging 2016;17:525-32.

23. Onishi T, Saha S, Ludwig D, et al. Feature tracking measurement of dyssynchrony from cardiovascular magnetic resonance cine acquisitions: comparison with echocardiographic speckle tracking. J Cardiovasc Magn Reson 2013;15:95

24. Orwat S, Kempny A, Diller G, et al. Cardiac magnetic resonance feature tracking: a novel method of assessing myocardial strain. Comparison with echocardiogra.phic speckle tracking in healthy volunteers and in patients with left ventricular hypertrophy. Kardiol Pol 2014;72:363-71.

25. Kempny A, Fernández-Jiménez R, Orwat $\mathrm{S}$, et al. Quantification of biventricular myocardial function using cardiac magnetic resonance feature tracking, endocardial border delineation and echocardiographic speckle tracking in patients with repaired tetralogy of Fallot and healthy controls. J Cardiovasc Magn Reson 2012;14:32.

26. Schuster A, Stahnke VC, Unterberg-Buchwald C, et al. Cardiovascular magnetic resonance feature-tracking assessment of myocardial mechanics: Intervendor agreement and considerations regarding reproducibility. Clin Radiol 2015;70:989-98.

27. Gertz RJ, Lange T, Kowallick JT, et al. Inter-vendor reproducibility of left and right ventricular cardiovascular magnetic resonance myocardial feature-tracking. PLoS One 2018; 13:e0193746. 